PROCEEDINGS OF THE

AMERICAN MATHEMATICAL SOCIETY

Volume 132, Number 6, Pages 1721-1728

S 0002-9939(04)07233-8

Article electronically published on January 20, 2004

\title{
ON THE EXISTENCE AND MULTIPLICITY OF POSITIVE SOLUTIONS FOR SOME INDEFINITE NONLINEAR EIGENVALUE PROBLEM
}

\author{
MANUEL DELGADO AND ANTONIO SUÁREZ
}

(Communicated by David S. Tartakoff)

\begin{abstract}
This paper is concerned with the existence, uniqueness and/or multiplicity, and stability of positive solutions of an indefinite weight elliptic problem with concave or convex nonlinearity. We use mainly bifurcation methods to obtain our results.
\end{abstract}

\section{INTRODUCTION AND MAIN RESULT}

In this work we analyze the positive solutions of the weight elliptic problem

$$
\begin{cases}\mathcal{L} u=\lambda m(x) f(u) & \text { in } \Omega, \\ u=0 & \text { on } \partial \Omega,\end{cases}
$$

where $\Omega$ is a bounded domain of $\mathbb{R}^{N}$ with regular boundary $\partial \Omega ; m \in C(\bar{\Omega})$ changes sign, $f: \mathbb{R}_{+} \mapsto \mathbb{R}$ satisfies some assumptions that will be detailed below, $\lambda \in \mathbb{R}$ will be regarded as a bifurcation parameter, and $\mathcal{L}$ is a second-order uniformly elliptic operator of the form

$$
\mathcal{L} u:=-\sum_{i, j=1}^{N} D_{i}\left(a_{i j}(x) D_{j} u\right)+\sum_{i=1}^{N} b_{i}(x) D_{i} u,
$$

with $a_{i j}=a_{j i} \in C^{1}(\bar{\Omega})$ and $b_{i} \in C^{1}(\bar{\Omega})$.

When $m \equiv 1$, (1.1) was treated in [2] and [9, showing that there exists at most one positive solution if $f$ is concave, but it may have multiple positive solutions when $f$ is convex.

When $m$ changes sign the study of (1.1) is more difficult. In order to state the results we need to introduce some notation. First, we can suppose without loss of generality that $\lambda>0$ (similar results are obtained if $\lambda<0$ ) and $f^{\prime}(0)=1$ (see Remark 1.2 where we show how to overcome this restriction). Since the principal eigenvalue of $\mathcal{L}$ is positive (observe that positive constants are supersolutions of

Received by the editors August 20, 2002 and in revised form, January 24, 2003.

2000 Mathematics Subject Classification. Primary 35J65; Secondary 35B32, 35P30.

Key words and phrases. Indefinite weight elliptic problem, nonlinear eigenvalue problem, bifurcation method.

The authors thank the Spanish Ministry of Science and Technology for research support under grant BFM2000-0797. 
$\mathcal{L}$, see [15]), it is well known (cf. [13]) that the linear eigenvalue problem with indefinite weight function

$$
\begin{cases}\mathcal{L} u=\lambda m(x) u & \text { in } \Omega, \\ u=0 & \text { on } \partial \Omega\end{cases}
$$

admits two principal eigenvalues (i.e., an eigenvalue having a positive eigenfunction) such that $\lambda_{-}<0<\lambda_{+}$.

When $\mathcal{L}=-\Delta, f(0)=0, f$ is concave, nonnegative and $f(1)=0$, Brown and Hess 6 proved that (1.1) possesses exactly one positive solution $(\lambda, u)$ such that $\|u\|_{\infty} \leq 1$ if $\lambda>\lambda_{+}$, and it has no positive solution if $\lambda \in\left[0, \lambda_{+}\right]$.

Recently, Brown and Ko [14] studied (1.1) when $f(u)=f_{1}(u)=u-u^{p}$ (concave) and $f(u)=f_{2}(u)=u+u^{p}$ (convex), $p>1$ (in fact, they consider a more general boundary condition). They showed that from the trivial solution $u=0$ bifurcates an unbounded in $\mathbb{R} \times C(\bar{\Omega})$ continuum (maximal closed and connected set) $\mathcal{C}$ of positive solutions at $\lambda=\lambda_{+}$. In the case $f=f_{1}$, the solutions $u \in \mathcal{C}$ satisfy $\|u\|_{\infty}<1$, and the projection of $\mathcal{C}$ onto $\mathbb{R}$ is $\left(\lambda_{+},+\infty\right)$. Moreover, for $\lambda \in\left(\lambda_{-}, \lambda_{+}\right) \backslash\{0\}$ and $p<(N+2) /(N-2)$ they proved the existence of a positive solution (different from the ones arising from bifurcation) by using variational methods.

In the convex case, $f=f_{2}, \mathcal{C}$ goes to the left. Assuming $p<(N+2) /(N-1)$ and some restrictions on $m$ in order to obtain a priori bounds for $\lambda \neq 0, \mathcal{C}$ approaches infinity as $\lambda \rightarrow 0^{+}$. They also proved that there is no positive solution in the particular case $\lambda=\lambda_{+}$, but did not provide information for $\lambda>\lambda_{+}$.

We generalize and improve these results in different ways. We consider a not necessarily selfadjoint operator and more general reaction function. In the case of concave $f$, we prove that the solutions obtained by variational methods in [14] constitute in fact a branch bifurcating from infinity at $\lambda=0$ whose projection on the $\lambda$ axis is $(0,+\infty)$, and we deduce the existence of a second positive solution for $\lambda \in\left(\lambda_{+},+\infty\right)$. On the other hand, when $f$ is convex, we show the existence of a positive solution if $\lambda \in\left(0, \lambda_{+}\right)$, and in the particular case $f=f_{2}$ we prove that there is no positive solution for $\lambda \geq \lambda_{+}$.

Specifically, we show the following result. Assume that

$$
\Omega_{ \pm}:=\left\{x \in \Omega: m^{ \pm}>0\right\}
$$

are open and regular sets, where $m^{ \pm}$represent the positive and negative part of $m$ respectively; and suppose that $m^{ \pm}(x) \approx\left[\operatorname{dist}\left(x, \partial \Omega_{ \pm}\right)\right]^{\gamma_{ \pm}}$for $x$ close to $\partial \Omega_{ \pm}$and some $\gamma_{ \pm} \geq 0$. Throughout this paper we are going to work with a smooth function $f: \mathbb{R}_{+} \mapsto \mathbb{R}, f(0)=0, f^{\prime}(0)=1$ and satisfying

$$
f^{\prime \prime}<0, \quad \lim _{s \rightarrow+\infty} \frac{f(s)}{s^{p}}=\alpha<0,
$$

or

$$
f^{\prime \prime}>0, \quad \lim _{s \rightarrow+\infty} \frac{f(s)}{s^{p}}=\beta>0,
$$

for some $p$ such that

$$
1<p<\min \left\{\frac{N+1+\gamma_{ \pm}}{N-1}, \frac{N+2}{N-2}\right\} .
$$

Our main result is the following. 


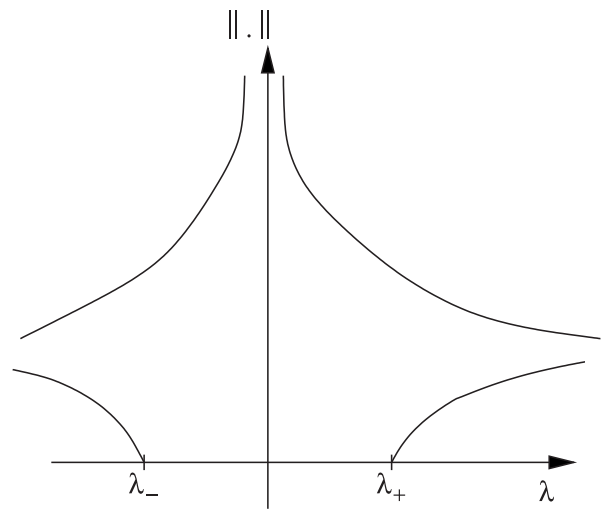

a)

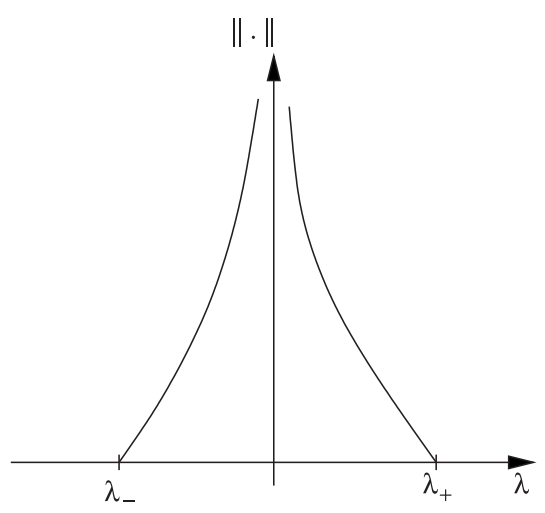

b)

FIGURE 1. Bifurcation diagram for (1.1)

Theorem 1.1. Suppose (1.4) holds.

a) Assume that $f$ satisfies $(f 1)$. Then (1.1) has a positive solution if and only if $\lambda \neq 0$. Moreover, for $\lambda \in\left(-\infty, \lambda_{-}\right) \cup\left(\lambda_{+},+\infty\right)$ there exist at least two positive solutions, one of them linearly asymptotically stable.

b) Assume that $f$ satisfies ( $f 2)$. Then (1.1) has a positive solution if $\lambda \in$ $\left(\lambda_{-}, 0\right) \cup\left(0, \lambda_{+}\right)$.

Furthermore, in any case for each sequence $\left(\lambda_{n}, u_{n}\right)_{n \in \mathbb{N}}$ of positive solutions of (1.1) such that $\lambda_{n} \rightarrow 0$, then $\left\|u_{n}\right\|_{\infty} \rightarrow \infty$ as $n \rightarrow \infty$.

See Figure 1, where we have summarized the information of this result.

Remark 1.2. As we said before, we can assume that $f^{\prime}(0)=1$. Indeed, if $f$ satisfies $(f 1)$ and $f^{\prime}(0)<0$, then (1.1) is equivalent to $\mathcal{L} u=\lambda m_{1}(x) h(u)$ with $m_{1}=-m$ and $h(u)=-f(u)$, and so it satisfies $(f 2)$. So, Theorem 1.1 b) applies in this case. On the other hand, if $f$ satisfies $(f 2)$ and $f^{\prime}(0)<0$, then (1.1) is equivalent to $\mathcal{L} u=\lambda m_{2}(x) j(u)$ with $m_{2}=-m$ and $j(u)=-f(u)$. In this case, we can apply Theorem 1.1 a).

In order to prove the above result, we include (1.1) in the more general equation

$$
\begin{cases}\mathcal{L} u=\mu m(x) u+\lambda m(x) g(u) & \text { in } \Omega, \\ u=0 & \text { on } \partial \Omega,\end{cases}
$$

with $\lambda$ fixed, $g(u)=f(u)-u$, adding $\mu \in \mathbb{R}$ as parameter and look for solutions to (1.5) for $\mu=\lambda$. This equation is interesting in itself, and it has attracted a great deal of attention recently (see, for example, [1], [3], [4, [5], 12] and [16]) when $m \equiv 1$ in the first term on the right-hand side of (1.5) and in [8] with the right-hand side of the form $\mu h(x) u+g(x) u^{p}$ and restrictive conditions on $h$ and $g$ that are not satisfied in our case. We give complete information on the bifurcation diagrams of (1.5), and deduce Theorem 1.1 from them.

An outline of the work is as follows. In Section 2 we study (1.5) when $f$ satisfies $(f 1)$ or $(f 2)$ and prove the main result of this paper. In the last section we study the particular case $f(u)=u+u^{p}$. 


\section{Study of the Equation (1.5)}

In this section we study the equation (1.5), giving a description of its bifurcation diagrams. We define $\Pi: \mathbb{R} \times C(\bar{\Omega}) \mapsto \mathbb{R}$, the projection map onto $\mathbb{R}$, i.e., $\Pi(\mu, u)=$ $\mu$.

Proposition 2.1. Assume [1.4], and fix $\lambda>0$.

a) If $f$ satisfies $(f 1)$, then (1.5) possesses a positive solution if $\mu>\lambda_{-}$. Moreover, from the trivial solution $u=0$ emanate two unbounded in $\mathbb{R} \times C(\bar{\Omega})$ continua of positive solutions $\mathcal{C}_{+}:=\left\{\left(\mu, u_{\mu}\right)\right\}$ and $\mathcal{C}_{-}:=\left\{\left(\mu, w_{\mu}\right)\right\}$ at $\mu=\lambda_{+}$and $\mu=\lambda_{-}$, respectively. Both continua bifurcate to the right, and $\Pi\left(\mathcal{C}_{-}\right) \supset\left(\lambda_{-},+\infty\right), \Pi\left(\mathcal{C}_{+}\right)=\left(\lambda_{+},+\infty\right)$. Finally, for $\mu>\lambda_{+}, u_{\mu}$ is linearly asymptotically stable and $u_{\mu} \neq w_{\mu}$.

b) If $f$ satisfies ( $f 2)$, then (1.5) possesses a positive solution if $\mu<\lambda_{+}$. Moreover, from the trivial solution $u=0$ emanate two unbounded in $\mathbb{R} \times C(\bar{\Omega})$ continua of positive solutions $\mathcal{C}_{+}:=\left\{\left(\mu, w_{\mu}\right)\right\}$ and $\mathcal{C}_{-}:=\left\{\left(\mu, u_{\mu}\right)\right\}$ at $\mu=\lambda_{+}$and $\mu=\lambda_{-}$, respectively. Both continua bifurcate to the left, and $\Pi\left(\mathcal{C}_{-}\right)=\left(-\infty, \lambda_{-}\right), \Pi\left(\mathcal{C}_{+}\right) \supset\left(-\infty, \lambda_{+}\right)$. Finally, for $\mu<\lambda_{-}, u_{\mu}$ is linearly asymptotically stable and $u_{\mu} \neq w_{\mu}$.

Proof. First, we prove part a). We begin by showing that if $(\mu, u)$ is a positive solution of (1.5) , then there exists $\mu_{0}<0$ (in fact, $\mu_{0}<\lambda_{-}$) such that

$$
\mu>\mu_{0}
$$

Since $(\mu, u)$ is a positive solution of (1.5), then $\lambda_{1}^{\Omega}\left(\mathcal{L}-\mu m-\lambda m \frac{g(u)}{u}\right)=0$, where $\lambda_{1}^{D}(\mathcal{L}+q)$ stands for the principal eigenvalue of $\mathcal{L}+q$ in a domain $D \subset \mathbb{R}^{N}$ with $q \in L^{\infty}(D)$ subject to homogeneous Dirichlet boundary conditions, and recall that $g(u)=f(u)-u$. Using the concavity of $f$ and the monotony properties of the principal eigenvalues with respect to the domain and the potential $q$, it follows that

$$
0=\lambda_{1}^{\Omega}\left(\mathcal{L}-\mu m-\lambda m \frac{g(u)}{u}\right)<\lambda_{1}^{\Omega_{-}}(\mathcal{L}-\mu m),
$$

and so (2.1) holds (see for instance Remark 6.3 in [15]).

Now, from the Crandall and Rabinowitz Theorem (cf. [10]) there exist $\varepsilon>0$ and two differentiable curves of positive solutions of the form $\left(\mu_{ \pm}(s), u_{ \pm}(s)\right)$ such that $\mu_{ \pm}(s)=\lambda_{ \pm}+\mu_{ \pm}^{1}(s)$ and $u_{ \pm}(s)=s\left(\varphi_{1}^{ \pm}+v_{ \pm}(s)\right)$ for $s \in(-\varepsilon, \varepsilon)$ with $v_{ \pm}(0)=0$, $\mu_{ \pm}^{1}(0)=0$ and where $\varphi_{1}^{ \pm}$are the positive eigenfunctions of (1.3) associated to $\lambda_{ \pm}$. Substituting these expressions in (1.5) and using that $\mathcal{L} \varphi_{1}^{ \pm}=\lambda_{ \pm} m(x) \varphi_{1}^{ \pm}$, we deduce that

$$
\left(\mathcal{L}-\lambda_{ \pm} m\right) v_{ \pm}(s)=\mu_{ \pm}^{1}(s) m(x)\left(\varphi_{1}^{ \pm}+v_{ \pm}(s)\right)+\lambda m(x)\left(\frac{f\left(u_{ \pm}(s)\right)}{s}-\varphi_{1}^{ \pm}-v_{ \pm}(s)\right),
$$

or equivalently,

$$
\frac{1}{ \pm \lambda_{ \pm}} v_{ \pm}-T_{ \pm} v_{ \pm}=\frac{1}{\lambda_{ \pm}} T_{ \pm}\left(\mu_{ \pm}^{1}(s)\left(\varphi_{1}^{ \pm}+v_{ \pm}(s)\right)+\lambda\left(\frac{f\left(u_{ \pm}(s)\right)}{s}-\varphi_{1}^{ \pm}-v_{ \pm}(s)\right)\right)
$$

where $T_{ \pm}: E:=C_{0}(\bar{\Omega}) \mapsto E ; T_{ \pm}:=(\mathcal{L})^{-1} M_{ \pm}$and $M_{ \pm}: E \mapsto E$ denotes the multiplication operator induced by the function $\pm m$. Considering $E$ ordered by its positive cone $P_{E}$, it is easy to see that $\varphi_{1}^{ \pm}$are the positive eigenfunctions of $T_{ \pm}$and that the spectral radius $r\left(T_{ \pm}\right)=r\left(T_{ \pm}^{*}\right)=\frac{1}{ \pm \lambda_{ \pm}}$, where $T_{ \pm}^{*}$ stands for the adjoint of 
$T_{ \pm}$in the sense of $L^{2}$. Denote by $\Phi_{ \pm}^{*} \in P_{E^{*}}$ the positive eigenfunctions of $T_{ \pm}^{*}$; then the Fredholm alternative applied to (2.2) lets us conclude that

$$
\lim _{s \rightarrow 0} \frac{\mu_{ \pm}^{1}(s)}{s}=-\lambda \frac{f^{\prime \prime}(0)}{2} \frac{\int_{\Omega}\left(\varphi_{1}^{ \pm}\right)^{2} \Phi_{ \pm}^{*}}{\int_{\Omega} \varphi_{1}^{ \pm} \Phi_{ \pm}^{*}}
$$

and so

$$
\operatorname{sign}\left(\lim _{s \rightarrow 0} \frac{\mu_{ \pm}^{1}(s)}{s}\right) \neq \operatorname{sign}\left(f^{\prime \prime}(0)\right) .
$$

Therefore, the bifurcation directions are both supercritical when $f$ is concave.

We now analyze the global behavior of these curves bifurcating from $\left(\lambda_{ \pm}, 0\right)$. By the Rabinowitz global bifurcation theorem (cf. [17) there exist two continua $\mathcal{C}_{+}:=\left\{\left(\mu, u_{\mu}\right)\right\}$ and $\mathcal{C}_{-}:=\left\{\left(\mu, w_{\mu}\right)\right\}$ of solutions bifurcating from $\left(\lambda_{+}, 0\right)$ and $\left(\lambda_{-}, 0\right)$, respectively. First, we study $\mathcal{C}_{+}$. By $(f 1)$, there exists $s_{\mu}>0$ such that $(\mu-\lambda) s_{\mu}+\lambda f\left(s_{\mu}\right)=0$, and for $s<s_{\mu}$ we have that $\lambda f(s)+(\mu-\lambda) s>0$. Consider the family $\bar{u}(\mu):=s_{\mu}$ of supersolutions of (1.5). Observe that $\bar{u}$ is not a solution and that for $\mu>\lambda_{+}$and close to it, $u_{\mu}<\bar{u}(\mu)$. Consequently, we can apply Theorem 2.2 in [1] to conclude that

$$
u_{\mu}<s_{\mu}
$$

for all $\mu \geq \lambda_{+}$. We are going to prove that $u_{\mu}$ is asymptotically stable, i.e., that

$$
\lambda_{1}^{\Omega}\left(\mathcal{L}-\mu m-\lambda m g^{\prime}\left(u_{\mu}\right)\right)>0
$$

Indeed, taking $\psi:=\lambda f\left(u_{\mu}\right)+(\mu-\lambda) u_{\mu}$, we have that $\psi>0$ by 2.5 and that

$$
\left(\mathcal{L}-\mu m-\lambda m g^{\prime}\left(u_{\mu}\right)\right) \psi=-\lambda f^{\prime \prime}\left(u_{\mu}\right) \sum_{i, j=1}^{N} a_{i j} D_{i}\left(u_{\mu}\right) D_{j}\left(u_{\mu}\right)>0,
$$

whence we obtain that $\psi$ is a strict supersolution of $\mathcal{L}-\mu m-\lambda m g^{\prime}\left(u_{\mu}\right)$, and hence (2.6).

It is clear now that $\mathcal{C}_{+}$is unbounded and that $\Pi\left(\mathcal{C}_{+}\right)=\left(\lambda_{+}, \infty\right)$. Indeed, at $\mu=\lambda_{+}$the direction is supercritical. By (2.6) this continuum can be prolonged indefinitely to the right (i.e., $\mathcal{C}_{+}$cannot bend back). Finally, by (2.5), if $\left(\mu, u_{\mu}\right) \in$ $\mathcal{C}_{+}$, then $u_{\mu}$ is bounded, and the above claim follows.

We now analyze $\mathcal{C}_{-}$. By (2.3) and (2.4), $\mathcal{C}_{-}$goes to the right at $\mu=\lambda_{-}$. By the Crandall and Rabinowitz theorem, $\mathcal{C}_{-}$does not reach $\left(\lambda_{+}, 0\right)$, because in a neighborhood of $\left(\lambda_{+}, 0\right), \mathcal{C}_{-}$entirely consists of $\left(\mu_{+}(s), u_{+}(s)\right), s>0$. On the other hand, by (2.6) it follows that in each neighborhood of $\left(\mu_{0}, u_{\mu_{0}}\right)$ in $\mathbb{R} \times C(\bar{\Omega})$ with $\mu_{0}>\lambda_{+}$ the unique positive solutions of (1.5) are of the form $\left(\mu, u_{\mu}\right)$ (see Proposition 20.6 in [2]), and so $\mathcal{C}_{-}$cannot finish in $\mathcal{C}_{+}$.

Finally, by (1.4) it follows from Theorem 4.3 in [3] that for $\mu$ in a compact interval of $\mathbb{R}$ the solutions $(\mu, u)$ of $(\mathbf{1 . 5})$ are bounded in $\mathbb{R} \times C(\bar{\Omega})$. So, $\Pi\left(\mathcal{C}_{-}\right)$is unbounded. This concludes the proof of part a).

Since the proof of part b) differs only slightly from the above one, we just sketch it. Let $(\mu, u)$ be a positive solution of (1.5). Since $f$ is convex it follows that

$$
0=\lambda_{1}^{\Omega}\left(\mathcal{L}-\mu m-\lambda m \frac{g(u)}{u}\right)<\lambda_{1}^{\Omega_{+}}(\mathcal{L}-\mu m),
$$




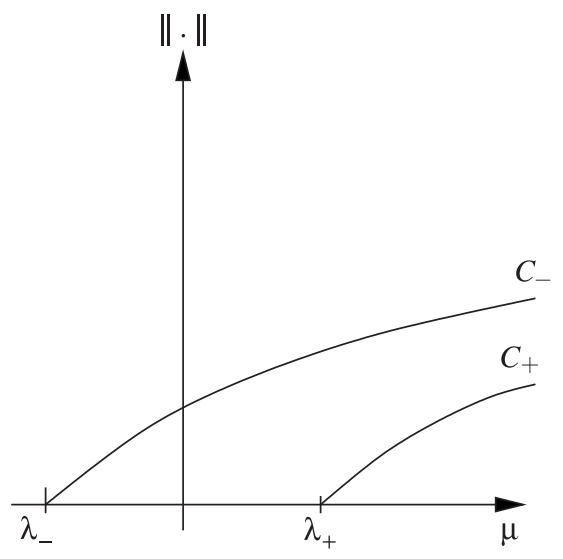

a)

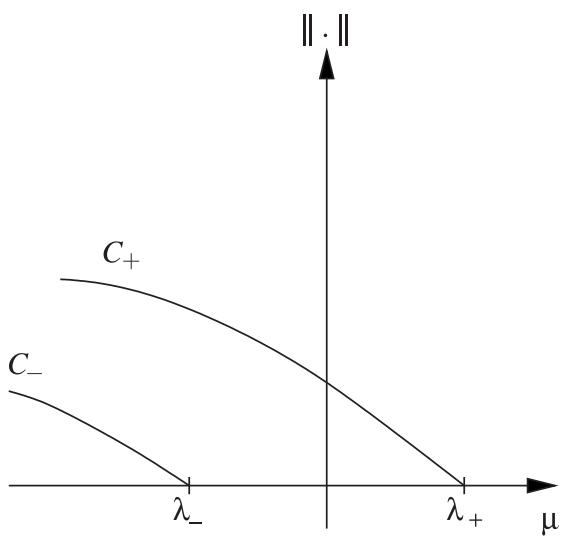

b)

Figure 2. Bifurcation diagram of (1.5)

and so there exists $\mu_{1}>0$ (in fact, $\mu_{1}>\lambda_{+}$) such that $\mu<\mu_{1}$.

By (2.3) and (2.4), we get that the bifurcation directions are in this case subcritical. On the other hand, by $(f 2)$ for negative $\mu$, there exists $t_{\mu}>0$ such that $(\mu-\lambda) t_{\mu}+\lambda f\left(t_{\mu}\right)=0$, and for $t<t_{\mu}$ we have that $\lambda f(t)+(\mu-\lambda) t<0$. With a similar reasoning to part a), we can prove that $u_{\mu}<t_{\mu}$, and, taking now as strict supersolution $\psi:=-\lambda f\left(u_{\mu}\right)+(\lambda-\mu) u_{\mu}$, we infer that $u_{\mu}$ is linearly asymptotically stable. The rest of the proof follows analogously.

Again, we have summarized the results of the above proposition in Figure 2.

We are ready to prove the main result.

Proof of Theorem 1.1. Consider $\lambda>0$ and $f$ satisfying $(f 1)$; the other case is similar. If $\lambda \in\left(0, \lambda_{+}\right]$, then there exists (see Figure $\left.2(\mathrm{a})\right)$ the solution $w_{\lambda}$ of (1.5) and so of (1.1). If $\lambda \in\left(\lambda_{+},+\infty\right)$, then there exist at least two positive solutions $w_{\lambda} \neq u_{\lambda}$, with $u_{\lambda}$ linearly asymptotically stable.

Assume now that there exists a sequence $\left(\lambda_{n}, u_{n}\right)_{n \in \mathbb{N}}$ of positive solutions with $\lambda_{n} \rightarrow 0$ and $\left\|u_{n}\right\|_{\infty} \leq K$ for some $K>0$. Since there does not exist a positive solution of (1.1) for $\lambda=0$, we obtain that $\left\|u_{n}\right\|_{\infty} \rightarrow 0$. We see that this is impossible. Indeed, we define

$$
w_{n}=\frac{u_{n}}{\left\|u_{n}\right\|_{\infty}} ;
$$

then $w_{n}$ is uniformly bounded and, by passing to a suitable sequence again denoted by $w_{n}$, we have $w_{n} \rightarrow w *$ as $n \rightarrow \infty$ for some $w \in C(\bar{\Omega})$ with $\left\|w^{*}\right\|_{\infty}=1$. But

$$
\mathcal{L} w_{n}=\lambda_{n} m(x) \frac{f\left(u_{n}\right)}{\left\|u_{n}\right\|_{\infty}},
$$

and so $\mathcal{L} w^{*}=0$, which is absurd. This completes the proof. 


\section{Remark 2.2.}

(1) Note that the existence of $\mathcal{C}_{+}$(resp. $\left.\mathcal{C}_{-}\right)$in the case $f$ concave (resp. convex) is independent of the value of $p$.

(2) Other conditions can be imposed on $p$ and $m$ to establish a priori bounds for the positive solutions of (1.5) for compact intervals of $\mathbb{R}$; see [3], [4] and [7.

\section{The Case When $\mathcal{L}$ Is Selfadjoint and $f(u)=u+u^{p}$}

In the particular case $f(u)=u+u^{p}$ and $b_{i} \equiv 0$ in (1.2), we can complete the information of Theorem 1.1 (b). Indeed, we are going to show that there exist positive solutions if and only if $\lambda \in\left(\lambda_{-}, \lambda_{+}\right)$. Let $\lambda \leq \lambda_{-}<0$ (the case $\lambda>\lambda_{+}$ can be treated analogously). Then it is well known that $\lambda_{1}^{\Omega}(\mathcal{L}-\lambda m) \leq 0$. Let $\varphi_{1}$ be the positive eigenfunction associated to $\mathcal{L}-\lambda m$, i.e.,

$$
\mathcal{L} \varphi_{1}-\lambda m \varphi_{1}=\lambda_{1}^{\Omega}(\mathcal{L}-\lambda m) \varphi_{1} .
$$

Multiplying this equation by $\varphi_{1}^{p}$ and using the Green identity, we get

$$
p \int_{\Omega} \varphi_{1}^{p-1} \sum_{i, j=1}^{N} a_{i j} D_{i}\left(\varphi_{1}\right) D_{j}\left(\varphi_{1}\right)=\lambda \int_{\Omega} m(x) \varphi_{1}^{p+1}+\lambda_{1}^{\Omega}(\mathcal{L}-\lambda m) \int_{\Omega} \varphi_{1}^{p+1},
$$

and so

$$
\int_{\Omega} m(x) \varphi_{1}^{p+1}<0
$$

Using Picone's inequality (see, for example, [5] or Lemma 4.1 in [16]), we obtain

$$
\int_{\Omega}\left(\frac{\varphi_{1}}{u}\right)^{p}\left[\mathcal{L} u \varphi_{1}-\mathcal{L} \varphi_{1} u\right]<0
$$

and hence

$$
\lambda \int_{\Omega} \varphi_{1}^{p+1} m(x)<\lambda_{1}^{\Omega}(\mathcal{L}-\lambda m) \int_{\Omega} \varphi_{1}^{p+1} / u^{p-1},
$$

which contradicts (3.1).

Remark 3.1. This same argument can be used to prove that 1.5 possesses a positive solution if and only if $\mu>\lambda_{-}$(resp. $\left.\mu<\lambda_{+}\right)$if $f=u-u^{p}$ (resp. $f=u+u^{p}$ ).

\section{REFERENCES}

1. S. Alama and G. Tarantello, On semilinear elliptic equations with indefinite nonlinearities, Calc. Var. Partial Differential Equations, 1 (1993), 439-475. MR 97a:35057

2. H. Amann, Fixed point equations and nonlinear eigenvalue problems in ordered Banach spaces, SIAM Rev., 18 (1976), 620-709. MR 54:3519

3. H. Amann and J. López-Gómez, A priori bounds and multiple solutions for superlinear indefinite elliptic problems, J. Differential Equations, 146 (1998), 336-374. MR 99e:35057

4. H. Berestycki, I. Capuzzo-Dolcetta, and L. Nirenberg, Superlinear indefinite elliptic problems and nonlinear Liouville theorems, Topol. Methods Nonlinear Anal., 4 (1994), 59-78. MR 96d:35041

5. H. Berestycki, I. Capuzzo-Dolcetta, and L. Nirenberg, Variational methods for indefinite superlinear homogeneous elliptic problems, NoDEA Nonlinear Differential Equations Appl., 2 (1995), 553-572. MR 96i:35033

6. K. J. Brown and P. Hess, Stability and uniqueness of positive solutions for a semi-linear elliptic boundary value problem, Differential Integral Equations, 3 (1990), 201-207. MR 90j:35009

7. W. Chen and C. Li, Indefinite elliptic problems in a domain, Discrete Contin. Dynam. Systems, 3 (1997), 333-340. MR 98a:35035 
8. S. Cingolani and J. L. Gámez, Positive solutions of a semilinear elliptic equation on $\mathbb{R}^{N}$ with indefinite nonlinearity, Adv. Differential Equations, 1 (1996), 773-791. MR 97e:35046

9. D. S. Cohen and T. W. Laestch, Nonlinear boundary value problems suggested by chemical reactor theory, J. Differential Equations, 7 (1970), 217-226. MR 41:3994

10. M. G. Crandall and P. H. Rabinowitz, Bifurcation from simple eigenvalues, J. Funct. Anal., 8 (1971), 321-340. MR 44:5836

11. J. L. Gámez, Sub- and super-solutions in bifurcation problems, Nonlinear Anal., 28 (1997), 625-632. MR 97j:35047

12. R. Gómez-Reñasco and J. López-Gómez, The effect of varying coefficients on the dynamics of a class of superlinear indefinite reaction-diffusion equations, J. Differential Equations, 167 (2000), 36-72. MR 2001k:35168

13. P. Hess and T. Kato, On some linear and nonlinear eigenvalue problems with an indefinite weight function, Comm. Partial Differential Equations, 5 (1980), 999-1030. MR 81m:35102]

14. B. Ko and K. J. Brown, The existence of positive solutions for a class of indefinite weight semilinear elliptic boundary value problems, Nonlinear Anal., 39 (2000), 587-597. MR 2000k:35098

15. J. López-Gómez, The maximum principle and the existence of principal eigenvalues for some linear weighted boundary value problems, J. Differential Equations, 127 (1996), 263-294. MR 97b:35037

16. J. López-Gómez, On the existence of positive solutions for some indefinite superlinear elliptic problems, Comm. Partial Differential Equations, 22 (1997), 1787-1804. MR 99m:35075

17. P. H. Rabinowitz, Some global results for nonlinear eigenvalue problems, J. Funct. Anal., 7 (1971), 487-513. MR 46:745

Dpto. de Ecuaciones Diferenciales y Análisis Numérico, Fac. Matemáticas, C/. Tarfia s/n, C.P. 41012, Universidad de Sevilla, Spain

E-mail address: madelgado@us.es

Dpto. de Ecuaciones Diferenciales y Análisis Numérico, Fac. Matemáticas, C/. Tarfia s/n, C.P. 41012, Universidad de Sevilla, Spain

E-mail address: suarez@us.es 